\title{
Endosonographic and elastographic features of a rare epidermoid cyst of an intrapancreatic accessory spleen
}

Accessory spleens are found in approximately $10 \%$ of the general population, of which $16 \%$ are intrapancreatic [1]. A previously healthy 49-year-old patient was referred to our tertiary center for further evaluation of a pancreatic mass. She had initially presented to another hospital with nonspecific abdominal pain. An abdominal computed tomography (CT) scan had revealed a solid mass in the tail of the pancreas. Endoscopic ultrasound-guided fine needle aspiration (EUS-FNA) was suggestive of a pancreatic neuroendocrine tumor (PNET).

At our institution, the patient had a normal physical examination with no lymphadenopathy, organomegaly, or a palpable abdominal mass. The patient was referred for EUS-guided tattooing to guide laparoscopic distal pancreatectomy. The EUS revealed a hypoechoic, $2.3-\mathrm{cm}$ mass in the tail of the pancreas. The mass was round and homogeneous, with well-demarcated and sharp borders, and a small cystic component ( $\bullet$ Fig. 1).

EUS-elastography showed inhomogeneous hardness as compared with the surrounding tissue ( Fig. 2 ).

EUS-guided tattooing was carried out by injecting $2.5 \mathrm{~mL}$ of sterile, purified carbon particle just proximal to the lesion as the needle was withdrawn to the surface of the pancreas ( $\bullet$ Fig. 3 ).

The patient subsequently underwent laparoscopic spleen-preserving distal pancreatectomy without complications. The tattoo was readily identified and demarcated a precise line of resection. Pathologic examination of the surgical specimen demonstrated a cystic mass within the pancreas. The mass had a well-defined capsule within which was splenic parenchyma and a small cyst lined by a layer of benign squamous epithelium. Pathologic diagnosis was consistent with an epidermoid cyst in an intrapancreatic accessory spleen (IPAS) ( $\bullet$ Fig. 4).

None of the 16 reported IPAS cases were diagnosed preoperatively as they are known to be difficult to clinically distinguish from other tumors. The value of EUS-FNA for their diagnosis needs further

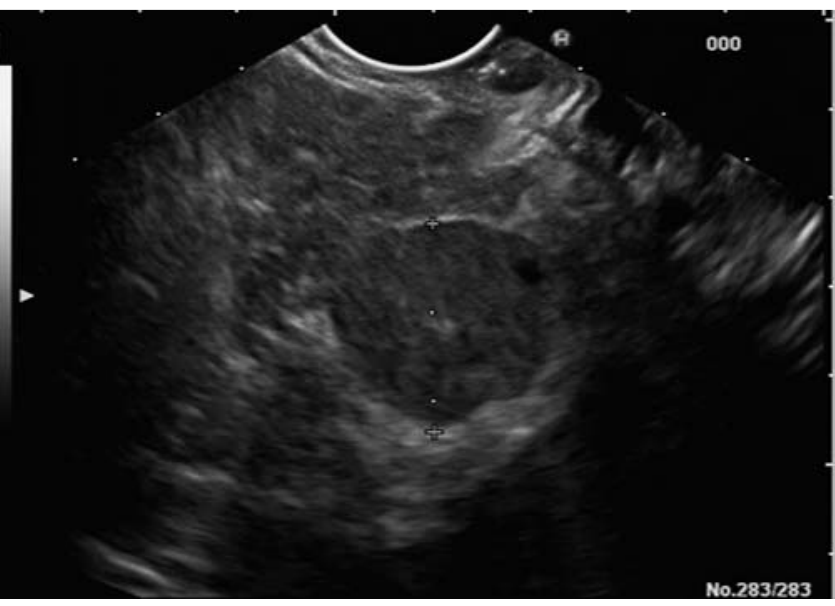

Fig. 1 Endoscopic ultrasound showing a hypoechoic, round, and well-circumscribed mass in the tail of the pancreas.

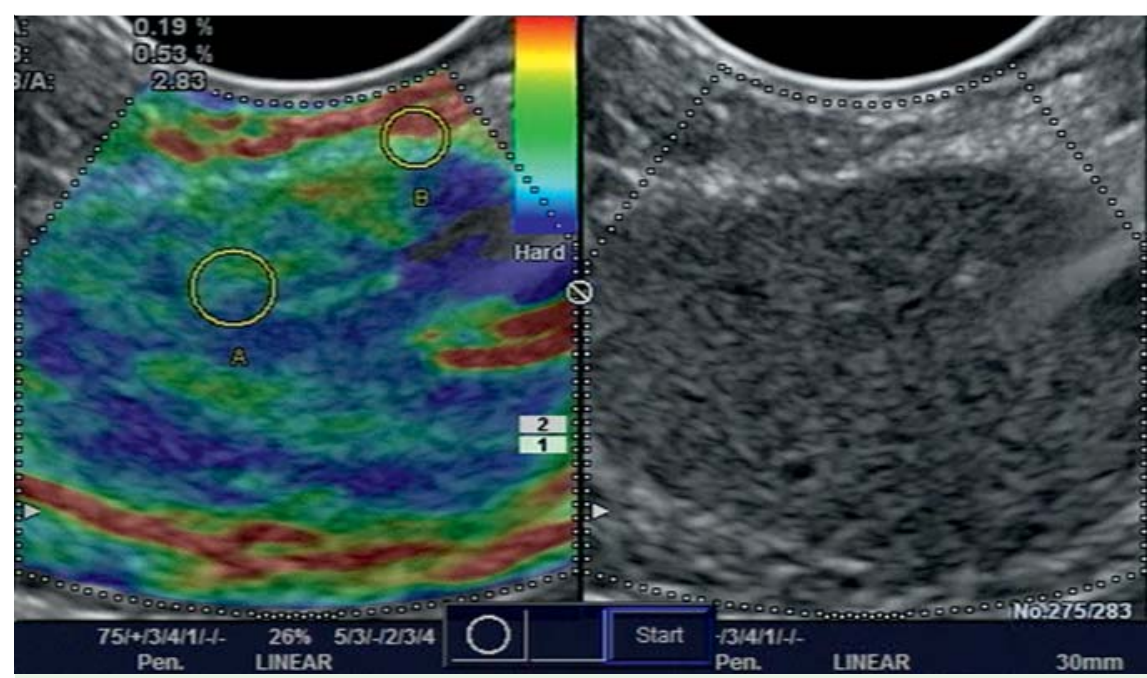

Fig. 2 Endoscopic ultrasound elastography showing inhomogeneous hardness (blue = significant hardness; green $=$ intermediate hardness).

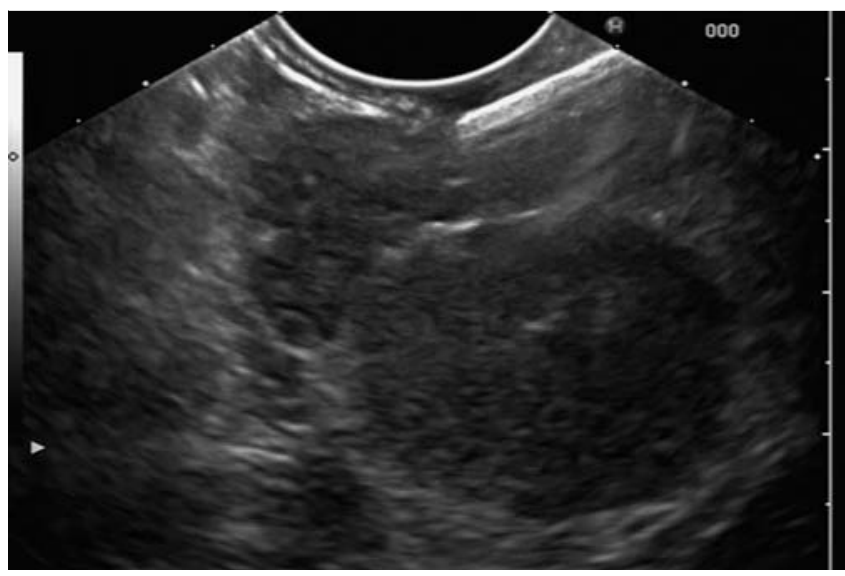

Fig. 3 Endoscopic ultrasound tattooing: injection of purified carbon particle just proximal to the mass as the needle is withdrawn to the surface of the pancreas.

study. FNA was not done in the current case because the lesion was presumed to 


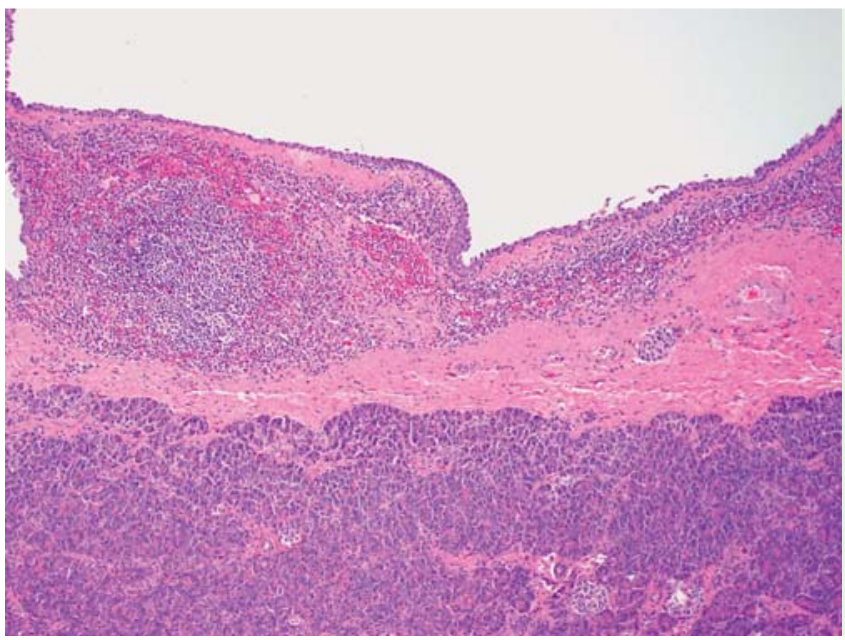
lining (top), the layer of

represent a PNET according to outside pathologic diagnosis.

Elastography is a means of measuring tissue stiffness. Malignant tissue is harder than benign tissue and elastography may be able to differentiate between them [2]. The system is set up to use a hue color map (red-green-blue), in which hard tissue areas are shown in dark blue, medium-hard tissue areas in cyan, intermediate hardness tissue areas in green, medium-soft tissue areas in yellow, and soft tissue areas in red [3]. In the current case, the epidermoid cyst demonstrated inhomogeneous hardness (mixture of blue and green). EUS-elastography has been used for the diagnosis of pancreatic cancer and malignant lymphadenopathy with variable sensitivity, specificity, and accuracy in different studies [3-5].

Endoscopy_UCTN_Code_CCL_1AF_2AZ_3AB

Competing interests: None
Fig. 4 Epidermoid cyst in an intrapancreatic accessory spleen. Note the thin epithelial splenic tissue, and the associated pancreatic parenchyma (bottom).

\section{References}

1 Halpert B, Alden ZA. Accessory spleens in or at the tail of the pancreas. A survey of 2700 additional necropsies. Arch Pathol 1964; 77 : $652-654$

2 Gao L, Parker KJ, Lerner RM et al. Imaging of the elastic properties of tissue - a review. Ultrasound Med Biol 1996; 22: 959-977

3 Iglesias-Garcia J, Larino-Noia J, Abdulkader I et al. EUS elastography for the characterization of solid pancreatic masses. Gastrointest Endosc 2009; 70: 1101-1108

4 Saftoiu A, Vilmann P, Ciurea T et al. Dynamic analysis of EUS used for the differentiation of benign and malignant lymph nodes. Gastrointest Endosc 2007; 66: 291 - 300

5 Saftoiu A, Vilmann P, Gorunescu F et al. Neural network analysis of dynamic sequences of EUS elastography used for the differential diagnosis of chronic pancreatitis and pancreatic cancer. Gastrointest Endosc 2008; 68: 1086 - 1094

M. A. Khashab ${ }^{1}$, M. I. Canto', V. K. Singh ${ }^{1}$, R. H. Hruban'², M. A. Makary ${ }^{3}$, S. Giday ${ }^{1}$

1 Department of Medicine and Division of Gastroenterology and Hepatology, Sol Goldman Pancreatic Cancer Research Center, Johns Hopkins Medical Institutions, Baltimore, Maryland, USA

2 Department of Pathology, Sol Goldman Pancreatic Cancer Research Center, Johns Hopkins Medical Institutions, Baltimore, Maryland, USA

3 Department of Surgery, Sol Goldman Pancreatic Cancer Research Center, Johns Hopkins Medical Institutions, Baltimore, Maryland, USA

\section{Bibliography}

DOI $10.1055 / \mathrm{s}-0030-1256272$

Endoscopy 2011; 43: E193-E194

(c) Georg Thieme Verlag KG Stuttgart · New York . ISSN 0013-726X

Corresponding author

S. Giday

Center for Digestive Health

1817 North Mills Avenue

Orlando

Florida 32803

USA

samgiday@gmail.com 